\title{
Postmenopausal Hormone Use and Secondary Prevention of Coronary Events in the Nurses' Health Study: A Prospective, Observational Study
}

\section{Citation}

Grodstein, Francine, JoAnn E. Manson, and Meir J. Stampfer. 2001. “Postmenopausal Hormone Use and Secondary Prevention of Coronary Events in the Nurses' Health Study: A Prospective, Observational Study." Annals of Internal Medicine 135 (1): 1. https:// doi.org/10.7326/0003-4819-135-1-200107030-00003.

\section{Permanent link}

http://nrs.harvard.edu/urn-3:HUL.InstRepos:41293016

\section{Terms of Use}

This article was downloaded from Harvard University's DASH repository, WARNING: This file should NOT have been available for downloading from Harvard University's DASH repository.

\section{Share Your Story}

The Harvard community has made this article openly available. Please share how this access benefits you. Submit a story. 


\title{
Postmenopausal Hormone Use and Secondary Prevention of Coronary Events in the Nurses' Health Study
}

\author{
A Prospective, Observational Study
}

Francine Grodstein, ScD; JoAnn E. Manson, MD; and Meir J. Stampfer, MD

Background: The Heart and Estrogen/progestin Replacement Study (HERS) was the first randomized clinical trial of combined hormone therapy and secondary prevention of coronary events. The trial had overall null results but reported an unexpected increased risk for recurrent events in the initial year, followed by a decrease during the final years.

Objective: To provide additional data on a time trend in risk for recurrent heart disease.

Design: A prospective, observational cohort study of secondary prevention of coronary heart disease.

Setting: Nurses' Health Study.

Patients: 2489 postmenopausal women with previous myocardial infarction or documented atherosclerosis; 213 cases of recurrent nonfatal myocardial infarction or coronary death were identified from 1976 through 1996.

Measurements: Information on hormone status and on recurrent disease was collected by using biennial questionnaires. Multi- variable-adjusted relative risks and $95 \% \mathrm{Cls}$ were calculated from logistic regression models.

Results: A trend of decreasing risk for recurrent major coronary heart disease events with increasing duration of hormone use was observed ( $P$ for trend $=0.002$ ). For short-term current users, the multivariate-adjusted relative risk for major coronary heart disease was $1.25(95 \% \mathrm{Cl}, 0.78$ to 2.00$)$ compared with never-users. However, after longer-term hormone use, the rate of second events was lower in current users than in never-users (relative risk, $0.38[\mathrm{Cl}, 0.22$ to 0.66$])$. No clear differences emerged between users of estrogen alone and users of estrogen combined with progestin. Overall, with up to 20 years of follow-up, the relative risk for a second event among current users of hormone therapy was $0.65(\mathrm{Cl}, 0.45$ to 0.95$)$ compared with never-users.

Conclusions: The risk for recurrent major coronary events seems to increase among short-term hormone users with previous coronary disease but to decrease with longer-term use.

Ann Intern Med. 2001;135:1-8.

www.annals.org For author affiliations, current addresses, and contributions, see end of text.
$\mathbf{T}_{\mathrm{s}}^{\mathrm{h}}$ he Heart and Estrogen/progestin Replacement Study (HERS) was a randomized clinical trial of postmenopausal hormone use in 2763 women (1). Unlike previous observational studies, it included only women with previous coronary heart disease, and hormone therapy was exclusively oral conjugated estrogen plus progestin. In HERS, overall rates of recurrent coronary heart disease did not differ between the treated and nontreated groups. However, in additional, unplanned analyses, the results of HERS were not uniformly null. A marked and statistically significant trend toward decreasing risk was observed with increasing duration of hormone use (1); in the first year, the rate of major coronary events was $52 \%$ higher in the treatment group. In the second year, rates were equal, but during the final fourth and fifth years, women assigned to hormone therapy had a $33 \%$ lower risk for coronary events.

The overall results of HERS and the apparent changes in risk over time were both unexpected. Since no previous investigations have examined the relation between duration of hormone use or combined hormone treatment to secondary prevention of coronary heart disease events, it remains unclear whether the time trend was authentic and whether the observations in HERS are limited to that hormone regimen (2).

In addition, we were interested in understanding how women's use of hormone therapy before their initial coronary disease event might affect the outcome of their hormone use after that event. In HERS, duration of hormone therapy before trial entry was necessarily disregarded (although 23\% of participants had previously taken hormones); thus, it remains unknown how, or whether, to account for hormone use or duration of use before a patient's initial coronary event.

To further examine the effects of duration of hormone therapy and to explore the effect of different hormone regimens, we investigated the relation between postmenopausal hormone use and secondary prevention of major coronary events in 2489 women with established coronary disease in the Nurses' Health Study, a large observational cohort. 
ARTICLE Postmenopausal Hormone Use and Recurrent Coronary Events

\section{Methods}

\section{The Nurses' Health Study Cohort}

The Nurses' Health Study began in 1976, when 121700 female nurses 30 to 55 years of age completed a mailed questionnaire about postmenopausal hormone use and medical history, including cardiovascular disease and its risk factors. Every 2 years, we mail follow-up questionnaires to the original participants to update information on risk factors and to identify newly diagnosed cases of major illnesses. The total follow-up for the cohort to date exceeds $92 \%$.

\section{Sample for Analysis}

For this analysis, we limited the cohort to postmenopausal women who reported a previous myocardial infarction or documented coronary atherosclerosis (coronary artery bypass graft surgery, percutaneous coronary revascularization, or angiographic evidence of $\geq 70 \%$ occlusion of one or more major coronary arteries). Such women were identified on the baseline questionnaire in 1976; postmenopausal women who reported one of these conditions on a subsequent follow-up questionnaire were added to the sample at that time. Women ranged in age from 34 to 73 years at entry into the sample for analysis; $60 \%$ of women had a natural menopause. These criteria closely parallel those used in HERS (1); however, in HERS, women with hysterectomy were excluded, occlusion was defined as at least $50 \%$, and participants ranged in age from 44 to 79 years.

We classified women as postmenopausal from the time of natural menopause or hysterectomy with bilateral oophorectomy. Women who underwent hysterectomy without bilateral oophorectomy were considered postmenopausal when they reached the age at which natural menopause had occurred in $90 \%$ of the cohort (54 years for smokers and 56 years for nonsmokers) (3). The women's reports of age at menopause and type of menopause were highly accurate (4).

In HERS, women with a history of breast or endometrial cancers were excluded. In the current study, we excluded women who reported stroke or cancer (except nonmelanoma skin cancer) on the 1976 baseline questionnaire at the outset and excluded women from further follow-up if they reported these diseases on a subsequent biennial questionnaire. This was done to avoid potential bias, in which major diseases such as stroke or cancer may have caused women to alter their hormone use and may be related to risk for coronary heart disease. This type of bias could not have occurred in a randomized trial, such as HERS.

Two hundred forty-eight postmenopausal women with coronary disease entered the analysis in 1976, and 2241 postmenopausal women were added during follow-up as they reported heart disease; thus, 17239 person-years accrued during up to 20 years of follow-up from 1976 to 1996.

\section{Ascertainment of Hormone Use}

In 1976, women were asked about current and past use and duration of hormone therapy; this information was updated on each biennial questionnaire. Beginning in 1978 and on each subsequent questionnaire, we collected information on type of hormone used. For the period 1976-1978, we assigned women to the type of hormone reported on the 1978 questionnaire. If no data were available on hormone therapy for a given 2-year period, those women were assigned to a missing category for that period. In the HERS trial, women who had taken hormones in the 3 months before their screening visit were excluded (although 23\% to $24 \%$ of the enrolled participants had previously used hormones). Because this study is observational, we could not impose a 3-month "washout" period; thus, we could not duplicate that aspect of the HERS protocol.

We analyzed duration of hormone use in two different ways. In the main analysis, we performed a "HERS replication" and used a method similar to that in HERS to calculate duration of use. In HERS, duration of hormone therapy began to accrue at randomization (regardless of previous hormone use); randomization occurred sometime after the participant's initial coronary disease event. Therefore, in our main analysis, we began accruing duration of hormone use for each participant immediately after her initial coronary disease event (regardless of hormone use before this event). For example, if a woman began hormone therapy in 1984 and had a revascularization procedure in 1986 , her duration of use in 1990 would be 4 years.

In an alternative analysis, we considered the women's full experience with hormone therapy (both before and after the initial coronary disease event). Thus, duration of hormone use accumulated continuously from 
start to termination of therapy. For example, if a woman began hormone therapy in 1984 and had a revascularization procedure in 1986, her duration of use in 1990 would be 6 years. Similar to HERS participants, 29\% of the nurses had taken hormones before their first coronary disease event.

\section{Identification of Second Coronary Disease Events}

Similar to HERS, in our study the category of "recurrent major coronary heart disease" included nonfatal myocardial infarction and fatal coronary disease that occurred between the return of the 1976 questionnaire and 1 June 1996. Nurses who reported a nonfatal infarction were asked for permission to review their medical records. Nonfatal myocardial infarctions were confirmed by hospital records if they met the World Health Organization criteria (5) (symptoms, plus either elevated cardiac enzyme levels or diagnostic electrocardiograms). Infarctions requiring hospitalization and corroborated by interview or letter, but for which medical records were unobtainable, were included as "probable."

Most deaths were reported by the participants' families. Every 2 years, we search the National Death Index (6) to identify deaths among nonrespondents; follow-up for death remains more than $98 \%$ complete to date. For all deaths possibly attributable to cardiovascular causes, we requested permission from relatives (subject to state regulations) to review the medical records. Deaths were considered due to coronary disease if medical records or autopsy findings confirmed a fatal myocardial infarction. Cases in which coronary disease was listed on the death certificate as the underlying cause of death and another, more plausible cause could not be discerned were included as "probable" cases, since the nurse was known to have had coronary disease before death. The investigators conducted all interviews and medical record reviews without knowledge of participants' hormone use status.

In separate analyses, results for probable cases of coronary disease $(20 \%)$ were almost identical to those for confirmed cases $(80 \%)$; thus, we present data from analyses in which confirmed and probable cases are combined.

\section{Statistical Analysis}

For each participant, person-months were allocated to hormone categories according to the baseline data and were updated every 2 years according to informa- tion received on follow-up questionnaires. Examination of type of hormone therapy was limited to users of oral conjugated estrogen with or without oral medroxyprogesterone acetate, as this was the most common hormone regimen. If information on hormone use was missing during a follow-up period, then person-time was assigned to a missing category for that time period. So that the study would be prospective, we established hormone status during each 2-year follow-up period from women's reports at the start of the time period; thus, we probably underestimate duration of use by an average of 1 year in both the main analysis and the alternate analysis. Follow-up for a participant ended with diagnosis of myocardial infarction, death, or 1 June 1996, whichever came first.

The analysis is based on incidence rates, using person-months of follow-up as the denominator. We used relative risk as the measure of association, defined as the incidence of second major coronary events among women in various categories of hormone use divided by the rate among women who never used hormones. We computed age-specific rates by using 5-year categories (7) and calculated age-adjusted relative risks by using Mantel-Haenszel rate ratios (8) with $95 \%$ confidence intervals (9).

We used pooled logistic regression across the ten 2-year periods to adjust simultaneously for potential confounding factors (10). In this approach, information obtained for each 2-year period is pooled for regression analysis as if it were a new observation, time-varying covariates are accommodated by assigning 2-year blocks of person-time to the covariate values at the start of each time period, and the dependence of incidence on time is modeled by using indicator variables. The distinction between pooled regression and the classic person-years approach is that we update risk factors at the beginning of each follow-up period. Nonetheless, this pooling yields a sample from which 2-year incidence can be examined and is equivalent to the person-years approach for generating relative risk estimates from Cox regression with time-dependent covariates (11). The necessary conditions for this equivalence include relatively short intervals and small probability of the outcome during each interval, both of which are satisfied here. Information on most variables was updated biennially, including age (5year categories), body mass index $\left(<21 \mathrm{~kg} / \mathrm{m}^{2}, 21\right.$ to 22 $\mathrm{kg} / \mathrm{m}^{2}, 23$ to $24 \mathrm{~kg} / \mathrm{m}^{2}, 25$ to $29 \mathrm{~kg} / \mathrm{m}^{2}, 30$ to 31

3 July 2001 Annals of Internal Medicine $\mid$ Volume 135 • Number $1 \mid \mathbf{3}$ 
ARTICLE Postmenopausal Hormone Use and Recurrent Coronary Events

\begin{tabular}{|c|c|c|c|}
\hline \multirow[t]{2}{*}{ Characteristic } & \multicolumn{3}{|c|}{ Hormone Use } \\
\hline & Never & Current & Past \\
\hline \multicolumn{4}{|l|}{ Parental myocardial infarction before } \\
\hline Hypertension, \% & 64.7 & 61.2 & 60.4 \\
\hline Diabetes mellitus, \% & 19.1 & 13.6 & 18.7 \\
\hline High serum cholesterol level, \% & 55.8 & 63.3 & 58.8 \\
\hline Current cigarette smoker, \% & 22.5 & 15.2 & 22.1 \\
\hline Mean age at entry, $y^{*}$ & 57.4 & 57.7 & 60.0 \\
\hline Mean age at menopause, $y$ & 48.4 & 45.0 & 45.0 \\
\hline Mean body mass index, $\mathrm{kg} / \mathrm{m}^{2}$ & 27.6 & 26.1 & 26.8 \\
\hline
\end{tabular}

* For mean age at entry, information on hormone use at entry was used.

$\mathrm{kg} / \mathrm{m}^{2},>31 \mathrm{~kg} / \mathrm{m}^{2}$ ), cigarette smoking (never; past; current smoker of 1 to 14 cigarettes/d, 15 to 24 cigarettes/d, $>24$ cigarettes/d), self-reported history of hypertension (yes or no), diabetes (yes or no), self-reported elevated cholesterol level (yes or no), and time period (ten 2-year periods). The following confounding variables were not updated: age at menopause $(<50$ years, 50 to 53 years, $>53$ years) and parental myocardial infarction before 60 years of age (yes or no). For certain analyses, saturated fat intake (quintiles), alcohol use (none, $<5 \mathrm{~g} / \mathrm{d}, 5$ to $14.9 \mathrm{~g} / \mathrm{d}, \geq 15 \mathrm{~g} / \mathrm{d}$ ), use of vitamin E supplementation (yes, no), multivitamin use (yes, no), aspirin use (none, 1 to 6 times/wk, $\geq 7$ times/wk), and physical activity (none or at least once per week) were added to the model. Information on these variables was updated every 4 years; follow-up was available from 1980 to 1996 only, because data on diet and exercise were first collected in 1980. We used SAS software (SAS Institute, Inc., Cary, North Carolina) for all analyses.

We calculated rate differences on the basis of the rate of recurrent major coronary heart disease events for postmenopausal Nurses' Health Study participants 60 to 69 years of age who never used hormone therapy. We multiplied this rate by the multivariate-adjusted relative risks to obtain the rates of recurrent coronary heart disease in various categories of hormone use. We subtracted the rates estimated for hormone use categories from the rate among women who never took hormones to obtain rate differences, or the number of recurrent coronary heart disease cases that could be attributed to or avoided with hormone use.

\section{Role of the Funding Source}

The present analysis was funded by the National Institutes of Health, which had no role in the study design, conduct or reporting of results.

\section{RESULTS}

We identified 138 nonfatal myocardial infarctions and 75 coronary deaths among women with previous myocardial infarction or coronary atherosclerosis. In women younger than 60 years of age who had never taken hormone therapy, the absolute rate of a recurrent major coronary event was 1389 per 100000 personyears; in women 60 to 75 years of age, this rate increased to 1678 per 100000 person-years. Never-users of hormones accounted for $38.6 \%$ of the follow-up time, past users accounted for $32.4 \%$, and current users accounted for $29.0 \%$. Of hormone therapy users, $53 \%$ used oral conjugated estrogen alone, $19 \%$ used oral conjugated estrogen plus oral progestin, and 28\% used other types of hormones (mostly oral estradiol or transdermal estrogen).

Current hormone users had a slightly better risk profile than past users or never-users (Table 1). Current users were less likely to have diabetes or to smoke cigarettes and had a somewhat lower mean body mass index compared with never-users. However, a higher proportion of current users reported a history of high cholesterol levels.

The age-adjusted relative risk for recurrent major coronary disease was 0.56 (95\% CI, 0.39 to 0.80 ) among current users of postmenopausal hormone therapy compared with never-users (Table 2). Adjustment for differences in age and other cardiovascular risk factors attenuated this relative risk somewhat to 0.65 (CI, 0.45 to 0.95 ), largely because current users had a lower prevalence of cigarette smoking and diabetes. However, controlling further for diet, vitamin supplement use, aspirin, and physical activity had no additional influence on the relative risk estimate (0.61 [CI, 0.39 to 0.96$])$; thus, we did not consider these factors in subsequent analyses because we would have had to limit follow-up (this information was first requested in 1980).

In the main analysis, when we examined duration of hormone use since the initial coronary disease event, we observed substantial differences in risk for a second event between short-term and longer-term hormone users (Table 2). We found an apparent 25\% increase in 
the risk for recurrent coronary heart disease events (relative risk, 1.25 [CI, 0.78 to 2.00]) among women with current use of less than 1 year compared with neverusers. Since we identified duration at the start of each 2-year follow-up period and probably underestimated duration of use during follow-up by an average of 1 year, this observed elevation in risk is probably lower than the true increase among short-term hormone users. However, longer-term hormone users experienced a decrease in risk for a second major coronary event (relative risk, 0.38 [CI, 0.22 to 0.66$]$ ) compared with neverusers. This trend of decreasing risk with increasing duration of current use was highly statistically significant ( $P=0.002$, using categories of $<1$ year, 1 to 1.9 years, and $\geq 2$ years of use).

Although we had limited statistical power to distinguish between the impact of different hormone regimens, we saw no strong evidence of varying effects for estrogen alone or combined with progestin; 11 of the 24 short-term users with coronary events $(46 \%)$ and 10 of the 18 longer-term users with coronary events (56\%) were taking oral conjugated estrogen alone. In addition, we found no substantial residual effects of hormone use after cessation of therapy: The multivariable-adjusted relative risk overall in past users was 0.80 (CI, 0.58 to 1.10).

To assess the absolute impact of hormone use on secondary prevention of coronary events, we calculated rate differences to measure the number of recurrent heart disease events that could be attributed to or avoided with postmenopausal hormone use. On the basis of these data from the Nurses' Health Study cohort and our current results, if 100000 postmenopausal women 60 to 75 years of age with heart disease received hormone therapy, one would expect 420 additional recurrent cases of coronary heart disease per year with short-term users but 1040 fewer cases per year after long-term use. However, since the rates of recurrent heart disease are much lower in this cohort of health professionals than in the general population, both of these rate differences probably underestimate the absolute impact of hormone therapy in the general population.

Finally, in an alternative analysis, we included duration of hormone use before each participant's first report of coronary disease to assess the effects of women's full experience with hormone therapy. In this alternative analysis, we still underestimated duration by an average of 1 year (as explained above); however, short-term use
Table 2. Risk for Major Coronary Heart Disease among Users and Nonusers of Postmenopausal Hormone Therapy in Women with Previous Myocardial Infarction or Coronary Atherosclerosis in the Nurses' Health Study (1976-1996)

\begin{tabular}{|c|c|c|c|c|}
\hline \multirow{3}{*}{$\begin{array}{l}\text { Hormone } \\
\text { Use }\end{array}$} & \multirow{2}{*}{ Follow-up } & \multirow{2}{*}{ Cases } & \multicolumn{2}{|c|}{ Relative Risk (95\% Cl) } \\
\hline & & & Age-Adjusted & $\begin{array}{l}\text { Multivariable- } \\
\text { Adjusted* }^{*}\end{array}$ \\
\hline & person-years & $n$ & & \\
\hline Never & 6652 & 99 & 1.0 (referent) & 1.0 (referent) \\
\hline Current† & 4997 & 42 & $0.56(0.39-0.80)$ & $0.65(0.45-0.95)$ \\
\hline$<1 y$ & 1549 & 24 & $1.06(0.67-1.66)$ & $1.25(0.78-2.00)$ \\
\hline $1-1.9 y$ & 1522 & 6 & $0.26(0.11-0.60)$ & $0.55(0.13-2.27)$ \\
\hline$\geq 2 y$ & 1926 & 12 & $0.38(0.20-0.71)$ & $0.38(0.22-0.66) \neq$ \\
\hline
\end{tabular}

* Adjusted for age, cigarette smoking, diabetes, high blood pressure, high cholesterol level, body mass index, age at menopause, parental history of premature myocardial infarction.

† Duration of use is underestimated by an average of 1 year because duration during each 2-year follow-up period is established at the start of each period.

$\ddagger P$ for trend $=0.002$. Test of trend was calculated by using categories of $<1$ year, $1-1.9$ years, or $\geq 2$ years of current hormone use.

was still largely limited to women who originally began hormone therapy after their first coronary disease event. In these short-term hormone users, we observed a more than twofold increased risk for recurrent coronary events (relative risk, 2.10 [CI, 0.88 to 5.01]) compared with never-users and an inverse relation after longer-term use (relative risk, 0.50 [CI, 0.32 to 0.77]). This trend of decreasing risk for recurrent coronary events with increasing duration of use since initiation of hormone therapy was statistically significant $(P$ for trend $=0.02$ ).

\section{Discussion}

In this prospective, observational study of secondary prevention of coronary events among 2489 postmenopausal women with a previous myocardial infarction or established atherosclerosis, we observed an apparent increase in the risk for a recurrent event with short-term hormone use. After longer-term use, however, the rate of second major coronary events seemed to decrease. A strong trend was observed in which risk for recurrent nonfatal myocardial infarction or coronary death decreased as duration of current hormone use increased. Although our sample size prohibited accurate analysis, we found no strong suggestion that risk was linked specifically to treatment with estrogen alone or in combination with a progestin.

A limitation of our study is that information on 3 July 2001 Annals of Internal Medicine $\mid$ Volume 135 • Number 1 |5 
ARTICLE Postmenopausal Hormone Use and Recurrent Coronary Events

hormones was self-reported, perhaps leading to some misclassification. However, we believe the reports to be accurate because participants are registered nurses with a demonstrated interest in medical research. Validation studies of numerous self-reported variables in this cohort, such as body weight (12) and diet (13), have proven that the nurses provide highly accurate information. In addition, reports of nonfatal myocardial infarction and coronary deaths were carefully documented. However, the observed elevation in risk among shortterm hormone users is probably lower than the true short-term increase, since we collect information on hormone use from the nurses at 2-year intervals and thus probably underestimated duration of use by an average of 1 year.

We began the Nurses' Health Study in 1976, when 248 postmenopausal women reported previous heart disease. Some of these women may have already experienced both a first and a second coronary event before 1976 and thus should not have been followed in the current analysis. However, women in the analysis ranged in age from 33 to 55 years at baseline in 1976; therefore, the probability that even a small proportion of these 248 women had already experienced two coronary events is very low. Moreover, in an analysis from which these 248 women with coronary disease at baseline were excluded, our results were consistent with those reported here, but because we had $20 \%$ fewer person-years of follow-up in the sample, we lost considerable statistical power.

The principal concern in our observational study is the possibility that women who choose to take hormones may differ from those who do not in ways that influence their risk for heart disease $(14,15)$. In particular, women whose initial coronary disease is more severe may be less likely to begin or to continue hormone therapy after their initial event than are women with less severe disease, leading to a "healthier user" bias. Such a bias might lead us to underestimate the true short-term elevation in risk and overestimate the true long-term decrease in risk. However, risk factor profiles were similar between current hormone users and never-users and between short-term and longer-term hormone users, in both our main "HERS replication" analysis and our alternative analysis (which included hormone use before the initial coronary disease event). For example, in the main analysis, $61.7 \%$ of short-term users and $61.4 \%$ of longer-term users reported hypertension, and $15.1 \%$ of short-term users and $15.4 \%$ of longer-term users were smokers. Furthermore, in all analyses, we carefully adjusted for differences in numerous risk factors; this adjustment produced only modest changes compared with our results adjusted for age alone. Thus, it is unlikely that confounding could completely explain our results.

In addition, we previously reported a twofold increase in the risk for pulmonary embolism (16) among current hormone users and a similar increase in the risk for cholecystectomy (17). Both estimates are consistent with those reported by HERS for these diseases (1), further confirming our ability to identify valid relations for hormone therapy in the Nurses' Health Study.

Few other studies have been published on secondary prevention of coronary events (18-24); in addition, none have been closely analogous to the HERS protocol, which examined only combination hormone therapy, or have distinguished between short-term and longer-term effects. Some investigators speculated that the HERS results were due to the combination therapy regimen used in that trial (2). Although we have limited statistical ability to separately examine the type of hormone used, data from our cohort of women largely taking oral conjugated estrogen alone suggest that this regimen may also be associated with a short-term increase in risk for a recurrent coronary event. In support of this observation, a 3-year randomized clinical trial of atherosclerosis progression in women with established heart disease (25) showed that therapy with neither estrogen alone nor estrogen with progestin resulted in a decrease of plaque area. In addition, all the observational studies (26) of combined hormone therapy and primary prevention of coronary heart disease (including our own [27]) have shown equivalent effects of both regimens.

The apparent impact of duration of hormone therapy in HERS was surprising. Our results on secondary prevention are consistent with the presence of a time trend in the risk for recurrent coronary events: When we examined duration of hormone use since initial heart disease (our main analysis) and duration since actual commencement of hormone therapy (our alternative analysis), both results suggested short-term adverse effects of hormone therapy. As explained above, the relative risk of 1.25 from our HERS replication is probably an underestimate of the true short-term risk but is consistent with the relative risk of 1.52 observed in HERS at 1 year of follow-up. However, in both our HERS 
replication and HERS, "short-term users" included women who may have been taking hormone therapy for many years before their initial heart disease event. Our relative risk of 1.25 and the HERS estimate of 1.52 are both lower than the relative risk of 2.10 from our alternative analysis, in which short-term hormone use largely represents women who first began therapy after their initial heart disease. Therefore, our data suggest that the risk among women with coronary disease may be marked by commencement of hormone therapy after their initial heart disease event; however, our confidence intervals are wide, and it is unlikely that many other studies will have adequate power to further investigate this issue. In one small study (28) of women taking hormone therapy for a mean of 8 years before receiving an intracoronary stent, target lesion revascularization and major adverse cardiac events were reduced over 3 years of follow-up in the women who continued taking hormones compared with those not taking hormones.

The cause of an apparent early increase in risk is unknown. An increase in venous thromboembolism among current hormone users was recently established $(1,16,29)$, and the HERS investigators suggested (1) that their results might be explained by a susceptible group of women who experience thromboembolic complications of hormone therapy in the short term (although no evidence links hormone use with risk for arterial thrombosis). Recent studies indicate that postmenopausal hormone use increases levels of C-reactive protein, an inflammatory marker (30); it is possible that inflammation contributes to plaque destabilization in vulnerable women. In contrast, however, experimental evidence has established an improved cholesterol profile (1) and enhanced vascular reactivity (30) in women with cardiovascular disease who take hormone therapy. Continued investigation is needed.

In contrast to the short-term increase in risk, we found a significant decrease in second coronary events for longer-term hormone users who were followed for up to 20 years. This finding is consistent with those of observational studies of secondary prevention (18-24), which have largely included patients who used hormones for a relatively long time and have all found lower risks for second cardiovascular events or death among women taking hormones. For example, O'Keefe and colleagues (21) reported better survival over 7 years among women with coronary angioplasty who contin- ued taking estrogen. In addition, the results from the fourth and fifth years of HERS suggested a potential reduction in second events for those assigned to hormone therapy (conditional on remaining event-free until year 4); this decrease is probably an underestimate because $25 \%$ of women had stopped taking their assigned hormone regimen by the third year of the trial. Although the overall HERS results were null, one can speculate about whether HERS may have yielded results similar to those of these observational studies if followup had been extended. However, extended follow-up of HERS may not be entirely generalizable since most participants were taking cholesterol-lowering medications; recent data from a small-scale randomized clinical trial suggest that hormone therapy alone may reduce progression of atherosclerosis, but estrogen in combination with lipid-lowering medication provides no benefits beyond that of lipid treatment (32).

In conclusion, results from HERS and the Nurses' Health Study, a large prospective, observational study, suggest that hormone therapy increases risk for a recurrent coronary event in the short term and that hormone therapy should not be initiated solely for prevention of recurrent heart disease. These studies also support the emergence of coronary benefits after several years of therapy. Additional data, including those from ongoing randomized clinical trials of primary and secondary prevention, will provide further information.

From Channing Laboratory, Brigham and Women's Hospital, Harvard Medical School, and Harvard School of Public Health, Boston, Massachusetts.

Acknowledgment: The authors thank Drs. Frank Speizer and Graham Colditz for their support.

Grant Support: By grants CA40356 and HL34594 from the National Institutes of Health. Dr. Grodstein is partially supported by grant AG13482 from the National Institute of Aging and a New Scholars Award from the Ellison Medical Foundation.

Requests for Single Reprints: Francine Grodstein, ScD, Channing Laboratory, 181 Longwood Avenue, Boston, MA 02115.

Current Author Addresses: Dr. Grodstein: Channing Laboratory, 181 Longwood Avenue, Boston, MA 02115.

Dr. Manson: Division of Preventive Medicine, Department of Medicine, Brigham and Women's Hospital, 900 Commonwealth Avenue East, Boston, MA 02215.

Dr. Stampfer: Department of Epidemiology, Harvard School of Public Health, 677 Huntington Avenue, Boston, MA 02115. 
ARTICLE Postmenopausal Hormone Use and Recurrent Coronary Events

Author Contributions: Conception and design: F. Grodstein, J.E. Manson, M.J. Stampfer.

Analysis and interpretation of the data: F. Grodstein, J.E. Manson, M.J. Stampfer.

Drafting of the article: F. Grodstein.

Critical revision of the article for important intellectual content: J.E. Manson, M.J. Stampfer.

Final approval of the article: F. Grodstein, J.E. Manson, M.J. Stampfer. Statistical expertise: F. Grodstein, M.J. Stampfer.

Obtaining of funding: J.E. Manson.

Administrative, technical, or logistic support: F. Grodstein, J.E. Manson, M.J. Stampfer.

Collection and assembly of data: F. Grodstein, J.E. Manson.

\section{References}

1. Hulley S, Grady D, Bush T, Furberg C, Herrington D, Riggs B, et al. Randomized trial of estrogen plus progestin for secondary prevention of coronary heart disease in postmenopausal women. Heart and Estrogen/progestin Replacement Study (HERS) Research Group. JAMA. 1998;280:605-13. [PMID: 9718051]

2. Herrington DM. The HERS trial results: paradigms lost? Heart and Estrogen/ progestin Replacement Study. Ann Intern Med. 1999;131:463-6. [PMID: 10498564]

3. Willett W, Stampfer MJ, Bain C, Lipnick R, Speizer FE, Rosner B, et al. Cigarette smoking, relative weight, and menopause. Am J Epidemiol. 1983;117: 651-8. [PMID: 6859020]

4. Colditz GA, Stampfer MJ, Willett WC, Stason WB, Rosner B, Hennekens $\mathrm{CH}$, et al. Reproducibility and validity of self-reported menopausal status in a prospective cohort study. Am J Epidemiol. 1987;126:319-25. [PMID: 3605058] 5. Organization WH: IHD Register. Report of the Fifth Working Group. Copenhagen: World Health Organization; 1971.

6. Stampfer MJ, Willett WC, Speizer FE, Dysert DC, Lipnick R, Rosner B, et al. Test of the National Death Index. Am J Epidemiol. 1984;119:837-9. [PMID: 6720679]

7. Rothman KJ, Boice JD Jr. Epidemiologic Analysis with a Programmable Calculator. Bethesda, MD: U.S. Department of Health, Education, and Welfare, Public Health Service, National Institutes of Health; 1979. NIH publication no. 79-1649.

8. Rothman KJ. Modern Epidemiology. Boston: Little, Brown; 1986.

9. Greenland S, Robins JM. Confounding and misclassification. Am J Epidemiol. 1985;122:495-506. [PMID: 4025298]

10. Cupples LA, D'Agostino RB, Anderson K, Kannel WB. Comparison of baseline and repeated measure covariate techniques in the Framingham Heart Study. Stat Med. 1988;7:205-22. [PMID: 3353604]

11. D'Agostino RB, Lee ML, Belanger AJ, Cupples LA, Anderson K, Kannel WB. Relation of pooled logistic regression to time dependent Cox regression analysis: the Framingham Heart Study. Stat Med. 1990;9:1501-15. [PMID: 2281238]

12. Rimm EB, Stampfer MJ, Colditz GA, Chute CG, Litin LB, Willett WC. Validity of self-reported waist and hip circumferences in men and women. Epidemiology. 1990;1:466-73. [PMID: 2090285]

13. Willett WC, Sampson L, Stampfer MJ, Rosner B, Bain C, Witschi J, et al. Reproducibility and validity of a semiquantitative food frequency questionnaire. Am J Epidemiol. 1985;122:51-65. [PMID: 4014201]

14. Barrett-Connor E. Postmenopausal estrogen and prevention bias. Ann Intern Med. 1991;115:455-6. [PMID: 1872493]

15. Matthews KA, Kuller LH, Wing RR, Meilahn EN, Plantinga P. Prior to use of estrogen replacement therapy, are users healthier than nonusers? Am J Epidemiol. 1996;143:971-8. [PMID: 8629615]

16. Grodstein F, Stampfer MJ, Goldhaber SZ, Manson JE, Colditz GA, Speizer FE, et al. Prospective study of exogenous hormones and risk of pulmonary embolism in women. Lancet. 1996;348:983-7. [PMID: 8855854]

17. Grodstein F, Colditz GA, Stampfer MJ. Postmenopausal hormone use and cholecystectomy in a large prospective study. Obstet Gynecol. 1994;83:5-11. [PMID: 8272307]

18. Henderson BE, Paganini-Hill A, Ross RK. Decreased mortality in users of estrogen replacement therapy. Arch Intern Med. 1991;151:75-8. [PMID: 1985611]

19. Kim SC, O'Keefe JH, Ligon RW, Giorgi LV, Cavero PG, Hartzler GO. Estrogen improves long-term outcome after coronary angioplasty [Abstract]. Circulation. 1995;92(Suppl 1):674.

20. Newton KM, LaCroix AZ, McKnight B, Knopp RH, Siscovick DS, Heckbert SR, et al. Estrogen replacement therapy and prognosis after first myocardial infarction. Am J Epidemiol. 1997;145:269-77. [PMID: 9012600]

21. O'Keefe JH Jr, Kim SC, Hall RR, Cochran VC, Lawhorn SL, McCallister BD. Estrogen replacement therapy after coronary angioplasty in women. J Am Coll Cardiol. 1997;29:1-5. [PMID: 8996287]

22. Sullivan JM, Vander Zwaag R, Hughes JP, Maddock V, Kroetz FW, Ramanathan KB, et al. Estrogen replacement and coronary artery disease. Effect on survival in postmenopausal women. Arch Intern Med. 1990;150:2557-62. [PMID: 2244772]

23. Sullivan JM, El-Zeky F, Vander Zwaag R, Ramanathan KB. Estrogen replacement therapy after coronary artery bypass surgery: effect on survival [Abstract]. J Am Coll Cardiol. 1994;23:49A.

24. Bush TL, Barrett-Connor E, Cowan LD, Criqui MH, Wallace RB, Suchindran $\mathrm{CM}$, et al. Cardiovascular mortality and noncontraceptive use of estrogen in women: results from the Lipid Research Clinics Program Follow-up Study. Circulation. 1987;75:1102-9. [PMID: 3568321]

25. Herrington DM, Reboussin DM, Brosnihan KB, Sharp PC, Shumaker SA, Snyder TE, et al. Effects of estrogen replacement on the progression of coronaryartery atherosclerosis. N Engl J Med. 2000;343:522-9. [PMID: 10954759]

26. Grodstein F, Stampfer M. The epidemiology of coronary heart disease and estrogen replacement in postmenopausal women. Prog Cardiovasc Dis. 1995;38: 199-210. [PMID: 7494882]

27. Grodstein F, Stampfer MJ, Manson JE, Colditz GA, Willett WC, Rosner $\mathrm{B}$, et al. Postmenopausal estrogen and progestin use and the risk of cardiovascular disease. N Engl J Med. 1996;335:453-61. [PMID: 8672166]

28. Khan MA, Liu MW, Singh D, Pal A, Chio FL, Lawson D, et al. Long-term (three years) effect of estrogen replacement therapy on major adverse cardiac events in postmenopausal women after intracoronary stenting. Am J Cardiol. 2000;86:330-3. [PMID: 10922445]

29. Daly E, Vessey MP, Hawkins MM, Carson JL, Gough P, Marsh S. Risk of venous thromboembolism in users of hormone replacement therapy. Lancet. 1996;348:977-80. [PMID: 8855852]

30. Cushman M, Legault C, Barrett-Connor E, Stefanick ML, Kessler C, Judd HL, et al. Effect of postmenopausal hormones on inflammation-sensitive proteins: the Postmenopausal Estrogen/Progestin Interventions (PEPI) Study. Circulation. 1999;100:717-22. [PMID: 10449693]

31. Rosano GM, Sarrel PM, Poole-Wilson PA, Collins P. Beneficial effect of oestrogen on exercise-induced myocardial ischaemia in women with coronary artery disease. Lancet. 1993;342:133-6. [PMID: 8101254]

32. Hodis HN, Mack WJ, Lobo RA, Shoupe D, Sevanian A, Mahrer PR, et al. Estrogen in the prevention of atherosclerosis trial (EPAT) [Abstract]. Circulation. 2000;102:2835. 